\title{
Soprannaturale e letteratura ("Il soprannaturale letterario. Storia, logica e forme di Francesco Orlando", Einaudi 2017)
}

\section{Gianni lotti}

\section{(2) OpenEdition Journals}

Édition électronique

URL : http://journals.openedition.org/studifrancesi/16134

DOI : 10.4000/studifrancesi. 16134

ISSN : 2421-5856

Éditeur

Rosenberg \& Sellier

\section{Édition imprimée}

Date de publication : 1 juillet 2019

Pagination : 126-129

ISSN : 0039-2944

\section{Référence électronique}

Gianni lotti, « Soprannaturale e letteratura ("Il soprannaturale letterario. Storia, logica e forme di Francesco Orlando", Einaudi 2017) », Studi Francesi [En ligne], 187 (LXIII | I) | 2019, mis en ligne le 01 avril 2020, consulté le 25 janvier 2021. URL : http://journals.openedition.org/studifrancesi/16134 DOI : https://doi.org/10.4000/studifrancesi.16134

\section{(c)}

Studi Francesi è distribuita con Licenza Creative Commons Attribuzione - Non commerciale - Non opere derivate 4.0 Internazionale. 


\section{Soprannaturale e letteratura \\ ("Il soprannaturale letterario. Storia, logica e forme di Francesco Orlando", Einaudi 2017)}

Abstract

The essay tackles the issue of the supernatural in literature as sketched by Francesco Orlando in his recently published posthumous study Il soprannaturale letterario. Storia, logica e forme. Orlando's proposal of reformulation and categorization of the theme - which is extremely innovative and exemplified by a range of texts belonging to an ample chronological span - is discussed in relation to the theoretical tenets of the author of Per una teoria freudiana della letteratura and takes into account the previous attempts of theorization of the supernatural.

Il libro postumo di Francesco Orlando sul soprannaturale - che ha potuto vedere la luce grazie all'ottimo lavoro svolto dai curatori Stefano Brugnolo, Luciano Pellegrini, Valentina Sturli sul materiale a disposizione costituito dagli appunti e dalla registrazione audio di un corso universitario tenuto dall'autore - affronta in apertura una questione metodologica che è stata sempre primaria nella teoria orlandiana del testo letterarario: quella del carattere organico e sistematico delle relazioni fra le varie componenti del testo stesso. Concezione di matrice strutturalista che, per la generazione a cui Orlando appartiene, implicava una presa di posizione critica nei confronti della visione crociana e idealistica di poeticità opposta a struttura. Coerentemente con questo assunto, fin dall'inizio l'accento viene posto sul problema delle regole di funzionamento del soprannaturale: non senza il piacere - in parte anche provocatorio, si può supporre - di sottolineare che le regole non solo presiedono al funzionamento del soprannaturale, e non ne sono affatto un motivo secondario, bensì che ne costituiscono un elemento poetico essenziale. Detto a proposito di Dante: «In tutta la Commedia la formulazione delle regole in vigore è spesso decisiva nel garantire la coerenza, gli snodi e perfino la vivacità narrativa del poema» (p. 10). E ancora: «Tutt'altro che meccaniche e astratte, di un'estrema funzionalità narrativa, le regole soprannaturali possono quindi situarsi al cuore dei momenti più commoventi del poema» (p. 11).

$\mathrm{Ma}$ - al di là della polemica latamente anti-crociana - mettere al centro le regole, per Orlando, significa assegnare la priorità a un problema conoscitivo. La dialettica fra una maggiore o minore disposizione ad attribuire credibilità a eventi o esseri in contrasto con il principio di realtà - fra «credito» e «critica» nei confronti del soprannaturale, secondo la sua espressione - traduce in effetti una questione legata alla conoscenza e alle cognizioni intellettuali del lettore. La domanda, almeno in prima istanza, non è tanto: cosa si prova di fronte al fenomeno soprannaturale? bensì: quali sono le opzioni cognitive che vengono mobilitate? L'effetto - e l'affetto - prodotto dalla rappresentazione letteraria del soprannaturale viene riportato a una varietà di aspetti costruttivi i quali, a loro volta, presuppongono il riferimento a un universo di convinzioni e credenze storiche che regolano i rapporti tra ciò che, in una determinata epoca, è ritenuto possibile o impossibile. In quanto il soprannaturale implica un problema di accettazione intellettuale della verosimiglianza dei contenuti, osserva Orlando, esso rappresenta un caso limite della questione più generale del tasso di 
illusione che è insito in ogni atto di lettura, il quale presuppone sempre, in qualche misura, la «volontaria sospensione dell'incredulità» di cui parla Coleridge.

L'operazione critica di Orlando, niente affatto scontata, consiste in una sovrapposizione tra soprannaturale $=$ dubbio intellettuale e soprannaturale $=$ esperienza affettiva. Ora, la decisione di tradurre, prioritariamente, l'effetto emotivo del soprannaturale in termini di cognizioni intellettuali si espone a un rischio di astrattezza (si veda, in tal senso, la posizione di Durkheim riportata a p. 20), dato che il concetto di soprannaturale implica quello di naturale in un senso che viene storicamente modulato in funzione dei diversi contesti culturali e che, di conseguenza, appare assai problematico decidere che cosa poteva essere credibile in un certo momento e che cosa no. Una simile obiezione è senz'altro seria, e infatti Orlando la prende molto seriamente. Tuttavia scatta qui la basilare opzione metodologica legata a Freud, che postula un'identificazione parziale tra filogenesi e ontogenesi, tra sviluppo storico collettivo e sviluppo individuale del bambino verso la condizione adulta, e quindi anche fra credenze intellettuali ed esperienze emotive. Dopo Freud - afferma Orlando - non ha molto senso parlare di opposizione tra razionalità e irrazionalità poiché ciò che è in gioco è una simultaneità fra differenti tipi di razionalità. E quindi, per ogni epoca, si dovrà parlare piuttosto di razionalità - ma anche di letteratura - compromissoria e compromessa col pensiero anti-logico o emotivo, con la costrizione esercitata dalla realtà sul pensiero «infantile» (p. 21) e con l'attrazione che quest'ultimo non cessa di suscitare nel pensiero adulto.

Il modello tipologico che Orlando costruisce a partire da questa idea centrale è però tutt'altro che avulso dalla storicità delle manifestazioni di cui è chiamato a rendere conto. C'è appena bisogno di ricordarlo: dal primo libro teorico del 1973, Per una teoria freudiana della letteratura, fino al libro sugli oggetti desueti uscito venti anni dopo (Gli oggetti desueti nelle immagini della letteratura. Rovine, reliquie, rarità, robaccia, luoghi inabitati e tesori nascosti, 1993 e 1994), la coniugazione del tipologico con lo storico ha rappresentato la grande originalità, e la grande forza, della proposta metodologica di Orlando. La forza di una sintesi - diciamo sbrigativamente - tra strutturalismo e storicismo (o meglio storicità del testo). E a proposito del rapporto fra i due termini, non è certo un caso che, nel libro, la prima serie di esempi di soprannaturale costruita in ordine cronologico preceda la serie costruita in un ordine tipologico, la quale ripropone i vari tipi di soprannaturale definiti induttivamente e rovescia in molti casi l'ordine cronologico: da un minimo di credito e un massimo di critica (soprannaturale di derisione), a un massimo di credito e un minimo di critica (soprannaturale di tradizione). Il risultato è un incrocio tra modellizzazione tipologica e prospettiva storica che apporta un formidabile arricchimento di senso alla nozione di soprannaturale letterario.

Per altro il capitolo III del libro (intitolato «Pluralità, limiti e ragioni del soprannaturale in letteratura») è uno straordinario saggio di storia letteraria che va molto al di là delle coordinate del soprannaturale in senso stretto e che è addirittura suscettibile di sviluppi antropologici - così come, del resto, molti altri progetti dell'ultimo Orlando rimasti incompiuti. La maggiore novità, in questo senso, consiste nella determinazione di una forma ottocentesca del soprannaturale letterario, detto di trasposizione, che si affianca al fantastico studiato da Tzvetan Todorov e che rivoluziona la configurazione della tematica del soprannaturale nel XIX secolo. Già negli esempi cronologici del capitolo I, di fronte al caso del Faust, opera nella quale i dialoghi possono essere apparentemente filosofici e astratti, Orlando aveva osservato «che qualcuno potrebbe chiedersi se questo Dio e questo diavolo siano esseri soprannaturali o pure ipostasi allegoriche» (p. 55), e aveva escluso la seconda possibilità, motivando la sua scelta in maniera estremamente convincente. La conclusione, in pagine che contano forse tra le più belle del libro, giungeva a leggere il soprannaturale di trasposizione in Goethe, con al centro una figura diabolica che rinvia alla negazione del passato e della storia, «soltanto in chiave di riferimento a un movimento di accelerazione storica, sconosciuto a tutte 
le epoche precedenti» (p. 62). E più precisamente, con un richiamo a Luckacs e non certo a Benjamin, in funzione di un'«allegoria del capitalismo» (p. 63) e del prodigioso dispiegamento della tecnica che ne ha accompagnato lo sviluppo: «E in ballo, in altri termini, proprio il potere del denaro, e a questo punto capiamo perché esso costituisca il nuovo soprannaturale moderno: perché proprio come la magia è in grado di conferire agli uomini forze e caratteristiche del tutto inaspettate» (p. 62).

Più in generale, questo aspetto del soprannaturale come «allegoria» di qualcosa che muta con le epoche e i contesti, questa varietà del potenziale allegorico del soprannaturale che si rinnova, è un elemento essenziale del discorso di Orlando in quanto dinamizza in maniera decisiva la rappresentazione e i significati del fenomeno. Di fronte alle varie forme di soprannaturale via via considerate, Orlando si interroga sempre sul fatto se si tratti o no, in effetti, di soprannaturale. Così, per esempio, nel caso del Don Chisciotte, egli si chiede se si possa parlare di soprannaturale a proposito di un testo il cui intento ideologico è precisamente quello di ridicolizzare la falsità dei romanzi di cavalleria e la mentalità magica che li sottende. In funzione delle diverse condizioni storico-culturali e delle capacità espressive individuali - sostiene il critico - un certo autore elaborerà «un tipo di soprannaturale radicalmente nuovo», cioè proporrà nella sua interpretazione del tema un diverso dosaggio delle relazioni fra presa sul serio e presa di distanze. Detto altrimenti, muteranno continuamente i rapporti di forza fra i termini della formazione di compromesso a cui la rappresentazione del soprannaturale è riconducibile.

In tal senso particolarmente interessante appare la formula del soprannaturale di indulgenza (di cui si parla nel cap. IV) che, da Ariosto a Voltaire, evolve verso quello detto di derisione. In esso, con un aumento cronologicamente progressivo della critica sul credito, il piacere consiste in un momentaneo accantonamento delle esigenze della ragione e in un abbandono «regressivo» a ciò che la nega o che comunque la mette in questione - con, in termini freudiani, lo spostamento da una rappresentazione che richiede maggiore dispendio psichico a una rappresentazione che richiede dispendio psichico minore. E ciò, per così dire, con l'alibi della coscienza che si tratta di un gioco più o meno polemico nei confronti di una credulità solo fuggevolmente accarezzata. Insomma, osserva Orlando, una ragione illuministica sempre più sicura di sé, della sua incommensurabile superiorità e della sua assoluta alterità rispetto alla logica ritenuta cattiva e manchevole del soprannaturale, può permettersi di indugiare nel gioco con un soprannaturale considerato retaggio di una mentalità superstiziosa: «...infatti ai suoi limiti ironici estremi l'Illuminismo è ancora capace di riconoscere, col linguaggio della logica che gli è propria, il fascino della logica che va sterminando» (p. 123). E in questo passeggero abbandono ludico si aprono spazi vitali per la conservazione della letteratura in quell'epoca, nella quale certe istanze di oltranzismo razionalistico - in Francia particolarmente forti da Saint-Évremond in poi - avrebbero addirittura preteso di abolirla. Così, per esempio, si dirà che Voltaire è un grande scrittore non perché se la prende con la mentalità religiosa e superstiziosa ma perché, sovvertendo quella mentalità, ne mima parodicamente il linguaggio, prestando in tal modo un credito sfuggente alla tradizione con una sorta di vampirizzazione retorica esercitata nei confronti della tradizione stessa. Al punto che si potrebbe concludere che una parte importante della letteratura illuministica - proprio quella più critica di Fontenelle, Montesquieu, Voltaire - esista paradossalmente come tale nella misura in cui si nutre di ciò che vuole distruggere.

Tornando al capitolo III, risulta illuminante a proposito del fantastico setteottocentesco, che Orlando chiama soprannaturale di ignoranza, lo sdoppiamento di questa categoria in funzione della risposta rispettiva alle domande «se» o «che cosa» - domande che, per altro, non si escludono vicendevolmente. Infatti, chiedersi, come avviene nel romanzo gotico di ascendenza illuministica, «se» qualcosa 
di soprannaturale è davvero avvenuto; oppure chiedersi, come avviene nel Giro di vite o in Horla di Maupassant (non citato, curiosamente, in tutto il libro), «che cosa» nasconde, a cosa allude, l'evento soprannaturale, sono due interrogativi molto diversi. Ora, secondo Orlando, la divaricazione fra i due tipi di domanda riflette una transizione capitale della Ragione europea: il passaggio da una tendenza a proiettare fuori di sé ciò che la mette in questione a una tendenza a problematizzare, e perfino a introiettare, ciò che è in apparenza razionalmente inspiegabile. Dunque, il passaggio da una ragione ancora dogmatica che si ritiene assoluta a una ragione già virtualmente freudiana che scava dentro se stessa provocando delle frane destinate a diventare sempre più profonde. Storicamente questo comporta delle implicazioni letterarie di straordinaria importanza, e ci riporta alla trasformazione di un soprannaturale ancora accreditato dalla religione e dalla mentalità diffusa a un soprannaturale, secondo Orlando, «assunto in proprio dalla letteratura». Se in epoca moderna, fino al momento dell'illuminismo compreso, è la Ragione assoluta a ergersi a giudice, e a considerare come scorie estranee al suo funzionamento il mito, la religione, la metafora (di cui, in vari modi, il soprannaturale può essere rappresentante), successivamente - a partire da Kant - la ragione si relativizza e, si potrebbe dire, diventa più comprensiva nell'atto stesso di realizzare la propria non assolutezza. In tal senso le riflessioni di Orlando su quella categoria «sdoppiata» che è il soprannaturale di ignoranza, risultano consonanti con la posizione di un grande storico dell'illuminismo, Peter Gay, il quale ha definito il Settecento non già il secolo del trionfo della ragione, secondo la formula corrente, bensì il secolo in cui la ragione moderna - quella cartesiana - comincia a entrare in crisi, ed è costretta a inglobare una serie di istanze (emotive, sentimentali, legate a reazioni e pulsioni cieche del corpo e all'esperienza sensoriale) che in precedenza era disposta a riconoscere solo negativamente, come non-ragione, come irrazionale.

In una prospettiva così ampia i fantasmi e i mostri, gli emblemi del soprannaturale, potranno venire concepiti non come il prodotto di un pensiero irragionevole, bensì per dirla di nuovo con Freud - come il prodotto di un pensiero inconscio, o - per dirla con Matte Blanco - come figure di un pensiero «simmetrico» intimamente e inestricabilmente intrecciato al pensiero razionale. E ciò, beninteso, non per concludere che il soprannaturale post-illuministico perde di consistenza, si psicologizza, diventa un tema spiegabile in termini di allucinazione o di follia, ma - al contrario - per affermare che esso può ricevere un nuovo fondamento ontologico, e una nuova concretezza letteraria, proprio grazie alla coscienza della debolezza costitutiva che la ragione va acquisendo. Così, la letteratura dell'Ottocento e del Novecento potrà legare il soprannaturale a una sotto-realtà, o surrealtà, o realtà altra inquietante quanto fascinosa - «Souvent dans l'être obscur habite un dieu caché» canta Gérard de Nerval nelle Chimères - o anche, come avviene in Goethe, alle istanze ideologiche e politiche che chiamano in causa gli imperativi utilitaristici del progresso borghese. Resta, come nota Orlando, che il funzionamento letterario del soprannaturale, dall'Ottocento in poi, si fonda su operazioni di trasposizione allegorico-referenziale in grado di motivarlo profondamente e di rinnovarlo, si tratti del coinvolgimento di referenti «storici, sociali, in qualche misura politici, e quindi databili» o di referenti «fissi, immobili e metastorici» (p. 158). Vale a dire che nella modernità più vicina a noi il soprannaturale letterario si dota di un potenziale simbolico inedito, relativamente indipendente dai suoi contenuti specifici tradizionali; un potenziale sostanziato dalla consapevolezza che il pensiero razionale funziona complementarmente a una serie di istanze che tendono continuamente a ribaltarne i fondamenti logici e a metterne in questione - con effetti più o meno perturbanti - le conquiste. 